\title{
Prussak's Space: Anatomy Revisited
}

\author{
${ }^{1}$ Department of Otolaryngology, Faculty \\ of Medicine, Mansoura University, \\ Mansoura, Egypt \\ ${ }^{2}$ Cleveland Clinic, Cleveland, $\mathrm{OH}, \mathrm{USA}$
}

Alaa A. Abou-Bieh, MD1, Thomas J Haberkamp, MD2

Abstract

Objectives:

1- Describe the anatomy of the Prussak's space (Ps) utilizing the recently available data due to the incorporation of endoscopes in its studying.

2- Evaluate the previously described theories about its development, physiology, aeration and pathophysiology of its diseases.

Methods:

Fifty-five temporal bones were dissected, and the anatomical details were studied utilizing an operating microscope and the otoendoscopes.

Results:

Being a 3D sagittal space located laterally and superficially to the tympanum and formed by mucosal and bony walls, four types of Ps could be distinct based on the pathways of their aeration and communication with the various spaces and areas of the tympanum as was demonstrated by the development of the mucosal folds, pouches and pathways. First type and the most common $(71 \%)$ was aerated solely retrogradely via the posterior pouch of von Troeltsch. Second type $(2 \%)$ was only aerated via the anterior pouch of von Troeltsch. Third type (2\%) was only aerated through a special superior mucosal pathway. Spaces classified under the fourth Ps type $(25 \%)$ were aerated through combined or multiple pathways of the above types.

Conclusions:

Ps unlikely to what has been usually thought, has different types. Although the differences between these types might look trivial, they mostly contribute majorly in the development and progress of the space pathological conditions. This might play a role in the explanation of the Ps isolated infections and also the mechanism of retraction pockets and cholesteatoma formation and the modes of their extension.

\section{Introduction}

The anatomy of Prussak's space (Ps) was described in 1867 by Ale xander Prussak when he published his studies on the anatomy of the human tympanic membrane. ${ }^{1}$ As most of other middle ear (ME) spaces, studying and dealing with this space as a separate compartment based mainly on the concept of the studying of various mucosal folds (MFs) attached to the ossicular chain with its ligaments and the tendons of the tensor tympani $\left({ }^{*}\right)$ and stapedius muscle $\left({ }^{* *}\right)$ along with the chorda tympani nerve (CTN). ${ }^{2}$ This concept and studies were pioneered by Helmholtz, 1868; von Troeltsch, 1881; Hammar, 1902;
Politzer, 1909; Balance and Green, 1919; Sobotta, 1920; Lowndes Yates, 1936; Maisonnet and Coudane, 1950.2 Another concept closely related to the mucosal folds (MFs) and middle ear (ME) compartmental studying was introduced by Chatellier and Lemoine in 1946, which is the epitympanic diaphragm made up of various membranous ligaments and folds (MFs) which, together with the malleus(M) and the incus (I), formed the floor of the epitympanic compartment and the theory of routes and pathways of tympanic ventilation. ${ }^{3}$ The problem with these mucosal folds, and therefore the concepts based on them, is that they are easily seen in the living and in fresh tem- 
poral bones (TB) and they disappear or disintegrate rapidly if the temporal bones (TB) are permitted to dry, frozen or immersed in fixative solutions and disintegrate in disease processes involving middle ear (ME) or are often considered as residues of inflammation or as adhesions. ${ }^{2}$ This problem together with the difficulty in documenting the ventilation pathways have led to the vague nature of this area and many conflicts about it the most prominent in them is the conflicting conclusions of the work of Proctor 2,4-6 and Palva ${ }^{7-13}$. The idea of utilizing different techniques in investigating middle ear (ME) compartmental anatomy and physiology has emerged. ${ }^{14-17}$ We aimed by our work to present the anatomical data obtained due to the incorporation of endoscopes as an untraditional method in its studying.

\section{Material and Methods}

The anatomy and variations were studied in 55 Fresh frozen temporal bones. To the best of our knowledge, all bones came from adult cadavers. There were 31 right temporal bones and 24 left. All bones were dissected by the same approach and all were studied utilizing an operating microscope and otoendoscopes with 00,300 and $70^{\circ}$ angles and 2.7 and $3 \mathrm{~mm}$ diameters. The photos were processed digitally by Photoswit ${ }^{\circledR}$ digital computer photo-system (Photoswit, USA).

\section{Results}

\section{Space boundaries:}

They were fixed in all specimens and in comparison to published literature.

- Medially: the neck (MN) of the malleus. - Inferiorly: the short process of the malleus (MSP).

- Superiorly (roof): the lateral malleolar fold (LMF) till the rim of the notch of Rivinus (NR).

- Anteriorly: the terminal flaring out of the annulus (AF) as it leaves the anterior tympanic spine (ATyS).

- Posteriorly: fibers of the annulus (AF) as it leave the posterior tympanic spine (PTyS) to end at the malleus neck with the posterior pouch of von Troeltsch (VTP) lies between the posterior malleolar fold (PMF) and the tympanic membrane (TM).

- Laterally: the Shrapnell's membrane (PF).

- The chorda tympani nerve (CTN) was laying $s$ in the free margin of the posterior malleolar fold (PMF) in all specimens.

Space position and orientation in relation to surroundings:

Ps had a fixed position in all our specimens and was usually a 3D sagittal space located laterally and superficially to the tympanum (in inferior position to the epitympanum and superior position to the mesotympanum).

Size:

Ps size in our specimens depended mainly on the length of the neck of the malleus. This was regardless the pneumatization of all the ME spaces and their type of development. The mean of the neck of the malleus (MN) length was $0.7 \mathrm{~mm}$ with difference $+/-0.4 \mathrm{~mm}$.

Ps aeration routes and communication pathways with other spaces were the most remarkable variable has been noticed regarding the Ps anatomy. Therefore, they were used to classify the Ps in the study specimens and also due to their proclaimed role in the ME pathophysiology. Four types of Ps could be distinct:

-First type: was the most common (39 TBs) (71\%) and the space was aerated solely retrogradely via the posterior pouch of von Troeltsch (VTP).

-Second type: (1 TB) (2\%) was aerated via the anterior pouch of von Troeltsch (vTA) only.

-Third type: (1 TB) (2\%) was aerated only through a special superior mucosal pathway in the lateral malleolar fold (LMF), mainly posteriorly between it and the lateral incudal fold (LIF).

-Fourth type: (14 TB) (25\%) were aerated through combined or multiple (1 specimen) pathways of the above types. 


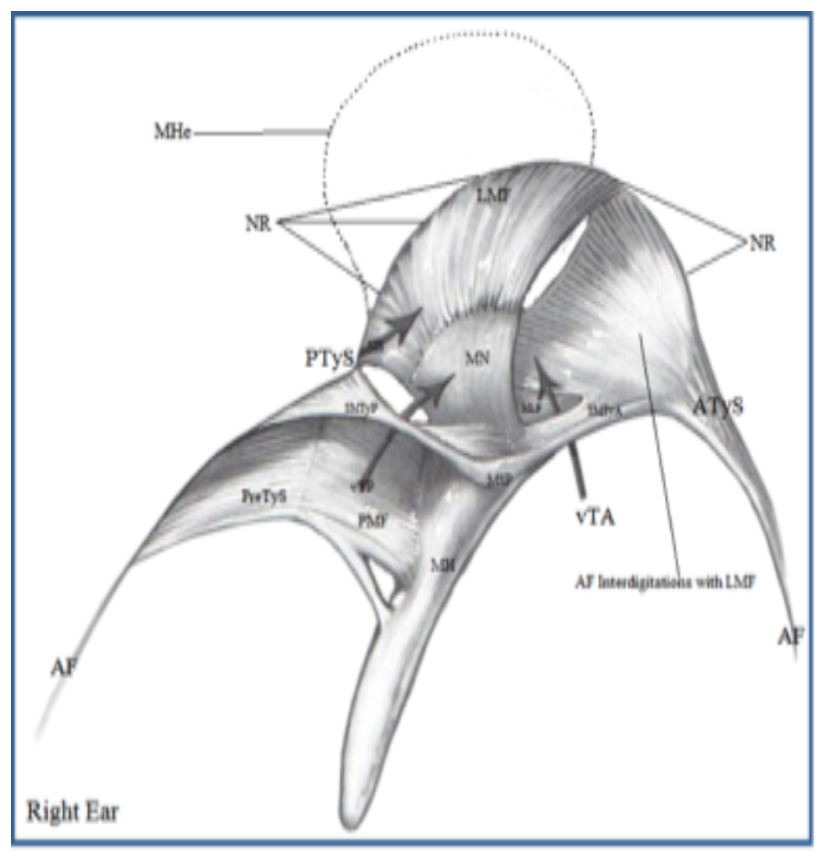

Figure 1. Ps: Outline \& Types

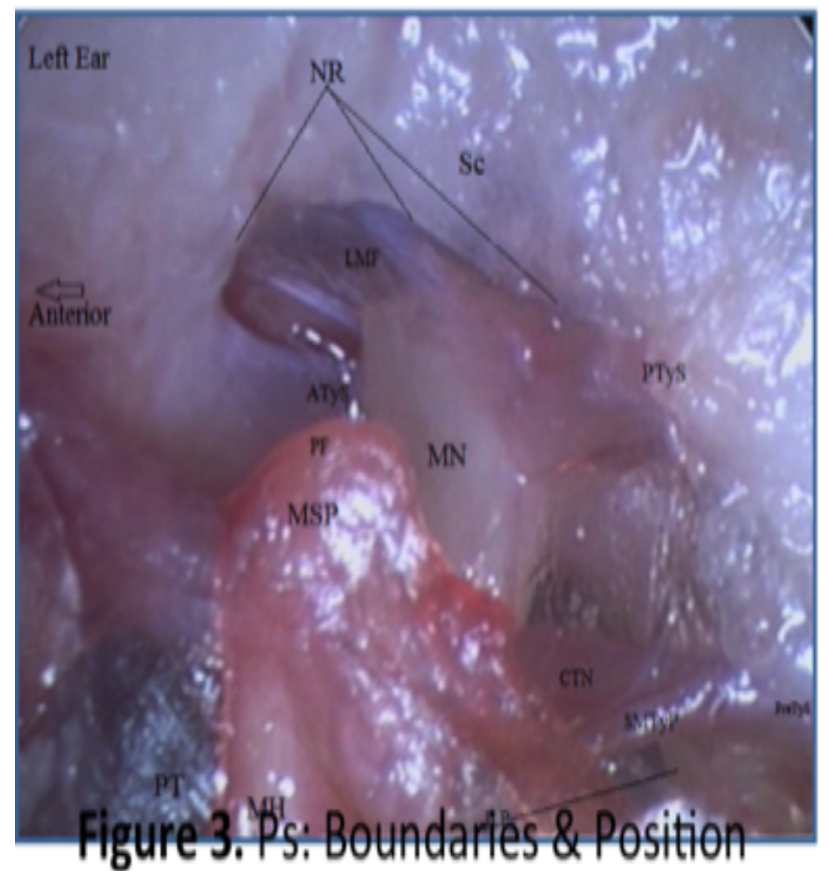

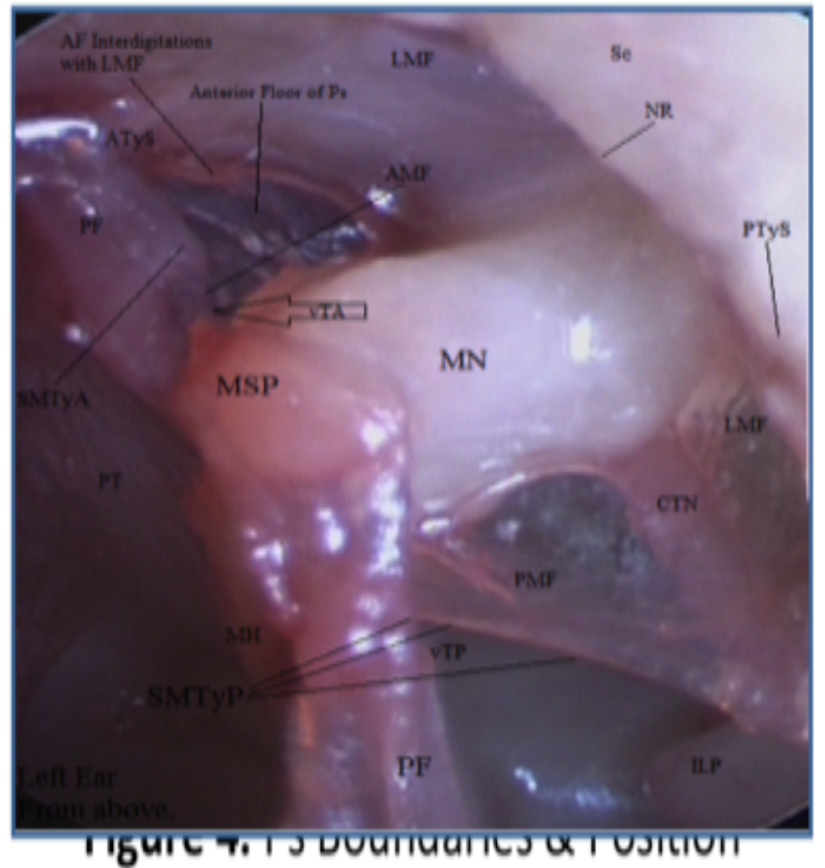

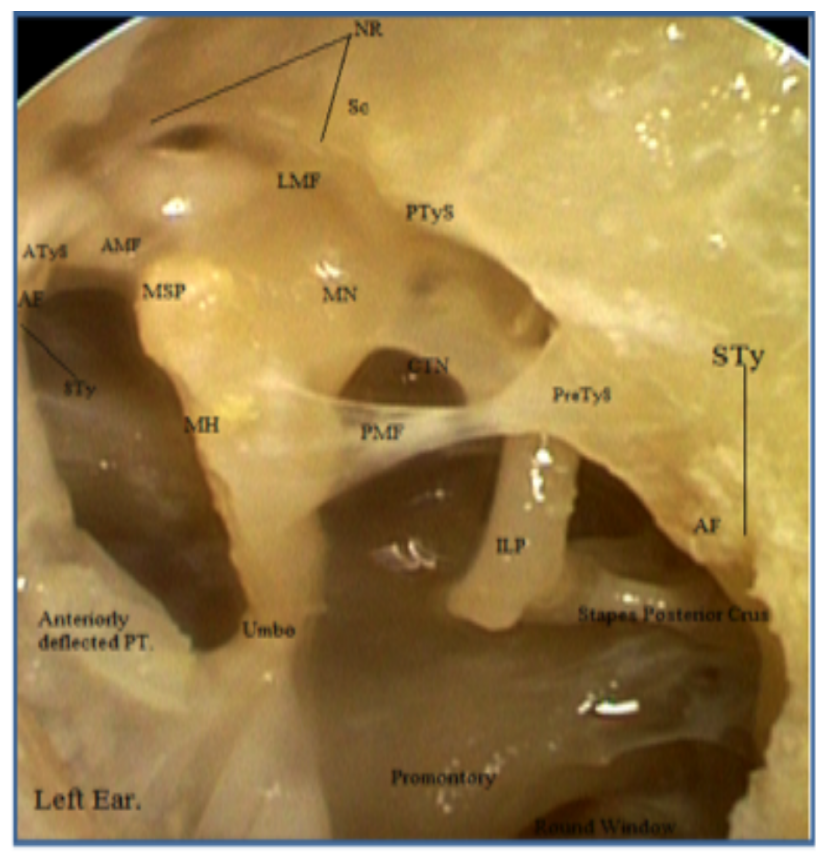

Figure 2. Ps: Outline

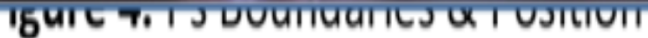




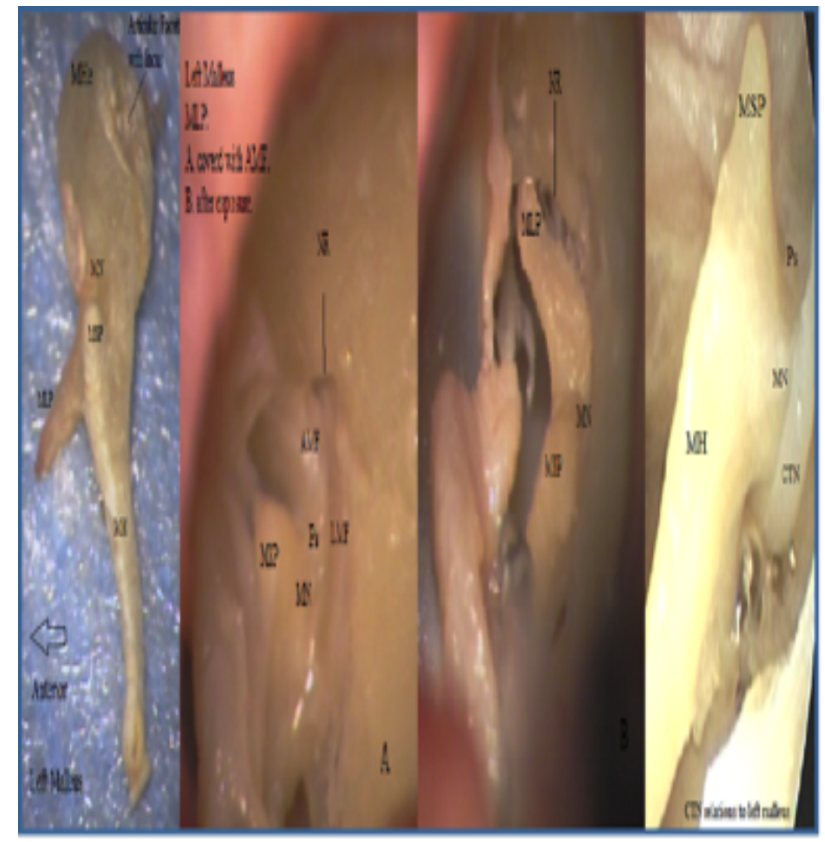

Figure 5. Ps: Malleus Relations

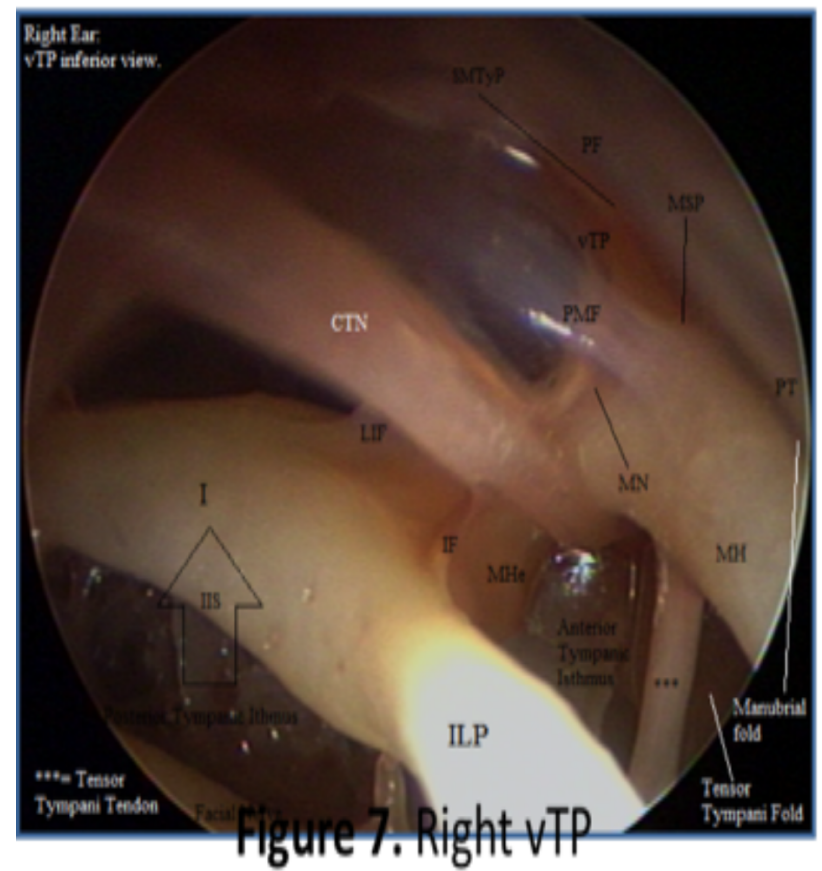

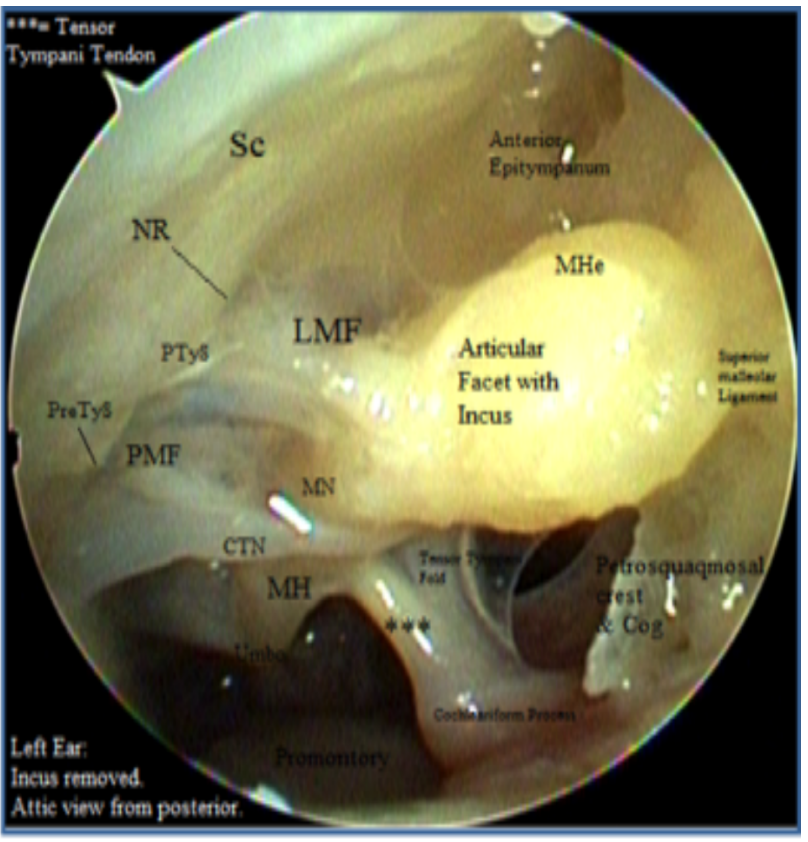

Figure 6. Ps: Attic View

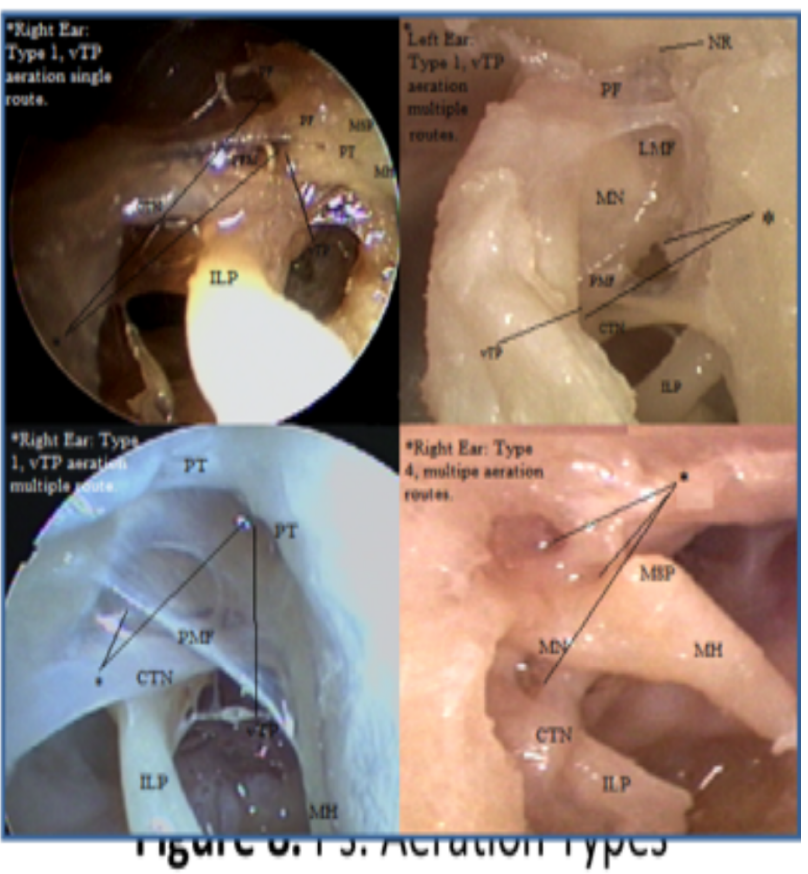




\section{Discussion}

The space lies between the notch of Rivinus $(\mathrm{NR})$, the anterior and posterior tympanic spines (A and PTyS) and the short process of the malleus (MSP). ${ }^{4}$ The medial and inferior aspects of Prussak's space (Ps) are formed, respectively, by the neck (MN) and the short process of the malleus (MSP). ${ }^{14}$ It is limited above by the lateral malleolar fold (LMF) which arises from the junction of the malleus head $(\mathrm{MHe})$ and neck $(\mathrm{MN})$ and radiates out to insert on the entire bony rim of the notch of Rivinus (NR) and medial wall of the scutum (Sc), thus forming a firm roof for Prussak's space (Ps) and the floor of the lateral malleolar space (LMS). 2,7,14 The space is limited anteriorly, and to some degree inferiorly, by the terminal flaring out of the annulus fibrosus (AF), the dense fibrocartilaginous ring to which the radial fibers of the drum attach, as it leaves the anterior tympanic spine (ATyS). As anteriorly when the annulus (AF) leaves the sulcus tympanicus (STy) it attach in part to the anterior tympanic spine (ATyS) then continues on as the stria membrana tympani anticus (SMTyA) to the short process (MSP), to radiate out to help form the floor of Prussak's space (Ps), to interdigitate with fibres of the lateral malleolar fold (LMF), and to attach to the bony rim of the notch of Rivinus (NR). ${ }^{2}$ Some authors $7-9,11,14$ describe the anterior aspect of the Prussak area (Ps) as being bounded by a very thin membranous fold among the tympanic membrane (TM) and the anterior malleolar ligament fold (AMF), which is inserted laterally onto the tympanic membrane (TM) and medially onto the neck of the malleus (MN) and the long process(MLP). Posteriorly, on leaving the posterior tympanic spine (PTyS), fibers of the annulus (AF) end at the malleus neck $(\mathrm{MN})$, forming the posterior floor of the Prussak area (Ps). As posteriorly when the annulus (AF) leaves the sulucs tympanicus (STy) its outer fibres insert on the posterior tympanic spine (PTyS) or extend in the stria membrana tympani posticus (SMTyP) to the short process of the malleus (MSP) while its inner fibres insert on the medially-placed pretympanic spine (PreTyS) or radiate out, forming the supporting structure for the posterior malleolar fold (PMF) and attaching on the postero-medial aspect of the upper third of the malleus handle $(\mathrm{MH}){ }^{2,4}$ Between the posterior malleolar fold (PMF) and the tympanic membrane (TM) lies the posterior pouch of von Troeltsch (vTP) which represents the Prussak's space (Ps) posterior wall.2,14 The lateral aspect is represented by the pars flaccid (PF) of the tympanic membrane (TM). 7,14

Beneath the floor of the attic (AT) and in the upper mesotympanum there are three compartments: the inferior incudal space (IIS) and the anterior (VTA) and posterior (VTP) pouches of von Troeltsch.2 The inferior incudal space (IIS) extends from the inferior surface of the incus (I) laterally to the posterior malleolar fold (PMF). It is limited medially by the medial incudal fold (MIF) and anteriorly by the interossicular fold (IF) which lies between the long crus of the incus (ILP) and the upper two thirds of the malleus handle $(\mathrm{MH})$. Between the posterior malleolar fold (PMF) and both the pars tensa (PT) and pars flaccid (PF) of the tympanic membrane (TM) lies the posterior pouch of von Troeltsch (vTP). The chorda tympani nerve (CTN) lies in the free margin of the posterior malleolar fold (PMF), although it may cross the posterior tympanum independent of this fold. The shallow anterior pouch of von Troeltsch (vTA) lies between that portion of the drumhead (TM) anterior to the malleus handle $(\mathrm{MH})$ and the anterior malleolar fold (AMF) which is draped on the anterior malleolar ligament (AML).

The posterior pouch of von Troeltsch (vTP) is the main route of ventilation 2,14 and as this pouch opens at the most cranial portion of the mesotympanum, so in most subjects, ventilation of Prussak's space (Ps) occurs through the communica- 
tion with the mesotympanum 2,12,14. Laterally and posterior in the attic (AT) the floor of the superior incudal space (SIS) is formed by the lateral malleolar fold (LMF) and by the lateral incudal fold (LIF) which extends posteriorly to the posterior incudal ligament (PIL). The entrance into Prussak's space (Ps) is usually located between the lateral malleolar fold (LMF) and the lateral incudal fold (LIF). ${ }^{2}$

Embryologically, the Prussak's space (Ps) develops from the medial saccule of the saccus medius which usually sends an offshoot forward between the lateral malleolar fold (LMF) and lateral incudal fold (LIF) to form Prussak's space (Ps). ${ }^{2}$

Approaching and visualization of the Prussak's space (Ps) in the current study was possible, safer and more convenient using endoscopes than the operating microscope. This has been highlighted in another published studies and confirmed in the current study. ${ }^{18}$

\section{Conclusions :}

Prussak's space (Ps) unlikely to what has been usually thought, has different types. Although the differences between these types might look trivial, they mostly contribute majorly in the development and progress of the space pathological conditions. This might play a role in the explanation of the Ps isolated infections and also the mechanism of retraction pockets and cholesteatoma formation and the modes of their extension.

\section{References}

1. Prussak A. Zur Anatomie des menschlichen Trommelfells. Arch Ohrenheilk 1867;3:255-78.

2. Proctor B. The development of the middle ear spaces and their surgical significance. J Laryngol Otol 1964;78:631-48.
3. Chatellier HP, Lemoine J. Le diaphragme interatticotympanique du nouveau-ne. Ann Otolaryngol Chir Cervicofac 1946;13:534-66.

4. Proctor B. Surgical anatomy of the ear and temporal bone. New York: Thieme; 1989. p. 75-8.

5. Proctor B. Attic-aditus block and the tympanic diaphragm. Ann Otol Rhinol Laryngol 1971;80 (3):371-5.

6. Proctor B. Epitympanic mucosal folds. Arch Otolaryngol 1971;94(6):578.

7. Palva T, Johnsson L. Epitympanic compartment surgical considerations: reevaluation. Am J Otol 1995;16:505-13.

8. Palva T, Ramsay H. Incudal folds and epitympanic aeration. Am J Otol 1996;17:700-8.

9. Palva T, Ramsay H, Bohling T. Tensor fold and anterior epitympanum. Am J Otol 1997;18:307-16.

10. Palva $\mathrm{T}$, Ramsey $\mathrm{H}$, Bohling $\mathrm{T}$. Lateral and anterior approach to supratubal recess and tensor fold. Am J Otol 1998;19:405-14.

11. Palva $T$, B?hling $T$, Ramsay $H$. Attic aeration in temporal bones from children with recurring otitis media: tympanostomy tubes did not cure disease in Prussak's space. Am J Otol 2000;21:485-493.

12. Palva T, Northrop C, Ramsey H. Aeration and drainage pathways of Prussak's space. Int J Pediatr Otorhinolaryngol 2001;57:55-65.

13. Palva T, Ramsey H. Aeration of Prussak's space is independent of the supradiaphragmatic epitympanic compartment. Otol Neurotol 2007;28:264-8. 
14. Marchioni D, Alicandri-Ciufelli M, Grammatica A, et al. Lateral endoscopic approach to epitympanic diaphragm and Prussak's space: a dissection study. Surg Radiol Anat 2010;32:843-52.

15. Kashiba K, Komori M, Yanagihara N, et al. Lateral orifice of Prussak's space assessed with a high-resolution cone beam 3dimensional computed tomography. Otol Neurotol 2011;32(1):71-6.

16. Neri E, Caramella D, Battolla L, et al. Virtual endoscopy of the middle and inner ear with spiral computed tomography. Am J Otol 2000;21(6):799-803.

17. Kirkpatrick K, Morris D, Wijhe R, et al. Demonstration of Different Aeration Pathways to Prussak's Space in the Human Epitympanum. Presented in 2006 ARO 29 annual midwinter research meeting.

18. Karchier EB, Niemczyk K, Or?owski A. Comparison of visualization of the middle ear by $\mathrm{mi}-$ croscope and endoscopes of $30^{\circ}$ and $45^{\circ}$ through posterior tympanotomy. Videosurgery Mininv 2014; 9(2): 276-281. 